\title{
Analysis of Decision-Feedback Based Broadband OFDM Systems
}

\author{
Alexandre de Baynast, Ashutosh Sabharwal and Behnaam Aazhang \\ Department of Electrical and Computer Engineering \\ Rice University, MS-366 - 6100 Main Street \\ Houston, Texas 77005 \\ Phone: +1 7133482359 \\ Fax: +1 7133486196 \\ Email: $\{$ debaynas, ashu, aaz $\}$ rice.edu
}

Technical Areas:

A. Communications Systems and Networking: 8. OFDM / Multicarrier;

B. Adaptive Systems and Processing: 1. Adaptive Filtering;

E. Signal Processing Algorithms and Applications: 1. DSP in Wireless Communications. 


\begin{abstract}
In wireless communications, about $25 \%$ of the bandwidth is dedicated to training symbols for channel estimation. By using a semi-blind approach, the training sequence length can be reduced while improving performance. The principle is as follows: the detected symbols (hard decision) are fed back to the channel estimator in order to re-estimate the channel more accurately. However, semi-blind approach can significantly deteriorate the performance if the bit error rate is high. In this paper, we propose to determine analytically the minimum Signal to Noise Ratio (SNR) from which a semi-blind method starts to outperform a training sequence based only system.
\end{abstract}

\section{INTRODUCTION}

${ }^{1}$ Spectral efficiency is a crucial issue in the wireless communications systems such as 4G, IEEE802.11n, 802.16. Approximatively $25 \%$ of the bandwidth is dedicated to training symbols for channel estimation. Semi-blind (SB) approaches have been proposed in order to reduce the training sequence length ([1],[2],[6]). The principle is as follows: the detected symbols (hard decision) are fed back to the channel estimator in order to re-estimate the channel more accurately. However, semi-blind approach can deteriorate the performance if the bit error rate is high. Therefore it is essential to determine the minimum Signal-to-Noise Ratio (SNR*) such that the semiblind approach outperforms a Training Sequence Based Only (TSBO) system for any SNR $>$ SNR*

The main difficulty to evaluate $\mathrm{SNR}^{*}$ is the detection stage which is a non linear function. Some authors have approximated the detector function by $\frac{2}{\pi} \arctan (x)$ [4]. However, this function remains highly non linear and the calculations for the closed-loop are barely intractable.

In this paper, we propose an elegant solution to determine analytically $\mathrm{SNR}^{*}$ : we express the channel re-estimate as a function of the probability of error of the symbols $P_{e}$. Since $P_{e}$ can easily be written as a function of the SNR of the system, we are able to determine analytically SNR*.

\section{SySTEM DESCRIPTION}

We consider a OFDM transmission for quasi-static fading channel. Assuming proper cyclic insertion and sampling, the OFDM system with $N_{c}$ subcarriers decouples the frequencyselective channel into $N_{c}$ flat-fading channels with the following input-output relation:

$$
r_{i}=h_{i} s_{i}+n_{i}, i=1, \ldots, N_{c},
$$

where $h_{i}$ is a complex channel spectrum coefficient. $s_{i}$ and $r_{i}$ are, respectively, the $M$-QAM transmitted signal and the received signal at the $i$-th subcarrier; $n_{i}$ is the i.i.d. zeromean additive noise with a variance $\sigma^{2}$. For each transmitted block of $N$ OFDM symbols, $N_{1}$ symbols are dedicated to the training whereas $N_{2}=N-N_{1}$ symbols forme the payload.

\footnotetext{
${ }^{1}$ This work was supported in part by NSF under grants ANI-9979465, EIA0224458, and EIA-0321266.
}

\section{LINEAR EQUALIZATION BASED ON TRAINING SEQUENCES ONLY}

In this section, we recall briefly main results [5] for zero-forcing linear equalization applied to a broadband (MIMO) OFDM system when TSBO approach is used.

The MMSE solution $\hat{h}_{i}$ for the channel estimation for each subcarrier $i, i=1, \ldots, N_{c}$ using a preamble of $N_{1}$ OFDM symbols is:

$$
\hat{h}_{i}=\frac{1}{N_{1}} \sum_{l=1}^{N_{1}} \frac{1}{\sigma_{s_{i}}^{2}} r_{i}[l] s_{i}^{*}[l]
$$

and the corresponding Zero-Forcing solution $\tilde{s}_{i}[l]$ for the estimated symbol $l$ for each subcarrier $i, i=1, \ldots, N_{c}$ is given by:

$$
\tilde{s}_{i}[l]=r_{i}[l] / \hat{h}_{i}
$$

Proposition 1: The probability of error per symbol for each subcarrier is bounded by ([5]):

$$
P_{e}^{T S B O} \leq 2 \operatorname{erfc}\left(\sqrt{\frac{3\left|h_{i}\right|^{2}}{4 \sigma^{2}\left(1+D_{M} / N_{1}\right)(M-1)}}\right)
$$

where $M$ is the modulation order and $D_{M}$ is the dispersion of the modulation.

\section{SEmi-Blind Linear Equalization}

In this section, we determine analytically the performance of the semi-blind channel estimation and equalization. The principle of a SB approach for channel estimation is as follows:

1) A short training sequence composed by $N_{1}$ symbols, is used at the beginning in order to get a rough channel estimation,

2) The first symbol of the payload data is estimated by using the channel estimation of the first step.

3) In order to improve the performance, we update the channel estimation by treating the previously detected symbols as training.

$N$ and $N_{2}$ denote the total length of a transmitted frame and the length of the payload, respectively: $N-N_{1}=N_{2}$. The semi-blind channel estimation $\hat{h}_{i}$ per subcarrier $i, i=$ $1, \ldots, N_{c}$ is:

$$
\hat{h}_{i}=\frac{1}{N_{1}+N_{2}}\left(\sum_{l=1}^{N_{1}} \frac{1}{\sigma_{s_{i}}^{2}} r_{i}[l] s_{i}^{*}[l]+\sum_{l=N_{1}+1}^{N_{1}+N_{2}} \frac{1}{\sigma_{\hat{s}_{i}}^{2}} r_{i}[l] \hat{s}_{i}^{*}[l]\right)
$$

Proposition 2: The probability of error per symbol and per subcarrier is bounded by:

$$
P_{e}^{S B} \leq 2 \operatorname{erfc}\left(\sqrt{\frac{3\left|h_{i}\right|^{2}}{4\left(\mu_{s b}^{2}+\sigma_{s b}^{2}\right)(M-1)}}\right)
$$

where $\mu_{s b}$ and $\sigma_{s b}^{2}$ are the bias and the variance of the estimation error, respectively. Their analytical expressions are given in Eqs. 7 and 8, respectively. Here, $P_{e}^{T S B O}\left(N_{1}\right)$ denotes the probability of symbol error by using TSBO approach with $N_{1}$ traning samples. $C_{1}(M)$ is a constant 


$$
\begin{gathered}
\mu_{s b}=h \cdot \frac{N_{2}}{N_{1}+N_{2}}\left[P_{e}^{T S B O}\left(N_{1}\right)\left(-1+C_{1}+C_{21}-C_{22}\right)+C_{22}\right] \\
\sigma_{s b}^{2}=\frac{\sigma^{2} D_{M}}{N_{1}+N_{2}}+\frac{|h|^{2} N_{2}}{\left(N_{1}+N_{2}\right)^{2}}\left\{\left[P_{e}^{T S B O}\left(N_{1}\right)\left(-1+C_{1}+C_{21}-C_{22}\right)+C_{22}\right]\left[P_{e}^{T S B O}\left(N_{1}\right)\left(-2+C_{1}+C_{21}-C_{22}+D_{M}^{\prime}\right)-2\right]\right\}(8) \\
C_{21}\left(M, P_{e}^{T S B O}\left(N_{1}\right),|h|\right)=\frac{2 D_{M} \exp \left(-5 d^{2}|h|^{2} / 4 \sigma^{2}\right) \sinh \left(d^{2}|h|^{2} / \sigma^{2}\right)}{\sqrt{\pi} \sigma^{2}[\operatorname{erf}(3 d|h| / 2 \sigma)-\operatorname{erf}(d|h| / 2 \sigma)]}
\end{gathered}
$$

where $d=\frac{\sqrt{6}}{(M-1)}$.

$$
C_{22}\left(M, P_{e}^{T S B O}\left(N_{1}\right),|h|\right)=\frac{8\left[(\sqrt{M}-2)+\exp \left(-d^{2}|h|^{2} / 8 \sigma^{2}\right) \sinh \left(d^{2}|h|^{2} / 8 \sigma^{2}\right)\right] \exp \left(-d^{2}|h|^{2} / 8 \sigma^{2}\right) \sinh \left(d^{2}|h|^{2} / 8 \sigma^{2}\right)}{M \sqrt{\pi} \sigma^{2}[1-\operatorname{erf}(d|h| / 2 \sigma)]}
$$

depending only of the modulation order. $C_{21}\left(M, P_{e}^{T S B O},\left|h_{i}\right|\right)$ and $C_{22}\left(M, P_{e}^{T S B O},\left|h_{i}\right|\right)$ are defined in Eqs. 9 and 10. Proofs will be provided in the full paper.

\section{COMPARISON BETWEEN THE TSBO AND THE SB APPROACHES}

Clearly, if the bias in the semi-blind approach is large, then the TSBO approach performs better. However, since the bias and the variance of the semi-blind estimator decreases linearly with the total frame length, better performance is expected for high SNRs.

Theorem 1: Assuming that the channel estimation error is Gaussian in both TSBO and SB schemes, SB outperforms TSBO if and only if:

$$
\log \sigma_{s b}^{2}+\frac{\mu_{s b}}{\sigma_{s b}^{2}} \geq \log \left(\frac{\sigma^{2} D_{M}}{N_{1}}\right)
$$

Therefore, we are able to determine the threshold $S N R^{*}=$ $1 / N_{c} \sum_{i}\left|h_{i}\right|^{2} /\left(\sigma^{2}\right)^{*}$ such that below this point, the semi-blind approach outperforms the approach based on training sequence only. The threshold $\left(\sigma^{2}\right)^{*}$ is solution of Eq. 11 when equality holds. It can be determined numerically with a fixed-point method.

\section{Simulation Results}

To illustrate the performance gain of SB over a TSBO approach, we simulate an OFDM transmission according to the IEEE802.16 standard [3]. Results are shown in Figs. 1 and 2 for 16-QAM and 64-QAM constellations, respectively. Analytical thresholds from Eq. 11 are: For 16-QAM, $S N R^{*}=$ $8.76,8.29$ and 8.37 in $\mathrm{dB}$ for $N_{1}=1,2,4$, respectively; For 64-QAM, $S N R^{*}=14.03 \mathrm{~dB}, 13.21$ and 12.63 in $\mathrm{dB}$ for $N_{1}=1,2,4$, respectively. The gap between the theoretical and simulated thresholds $(0.5-1 \mathrm{~dB})$ is essentially due to the upper bound of the probability of symbol error which is not tight at low SNR. Clearly, SB outperforms TSBO as soon as SER is small enough for any training sequence length and modulation order.

\section{CONCLUSION}

In this paper, we determined analytically the threshold SNR* from which Semi-Blind Approach outperforms Training Sequence Based Only method. Simulations validate our

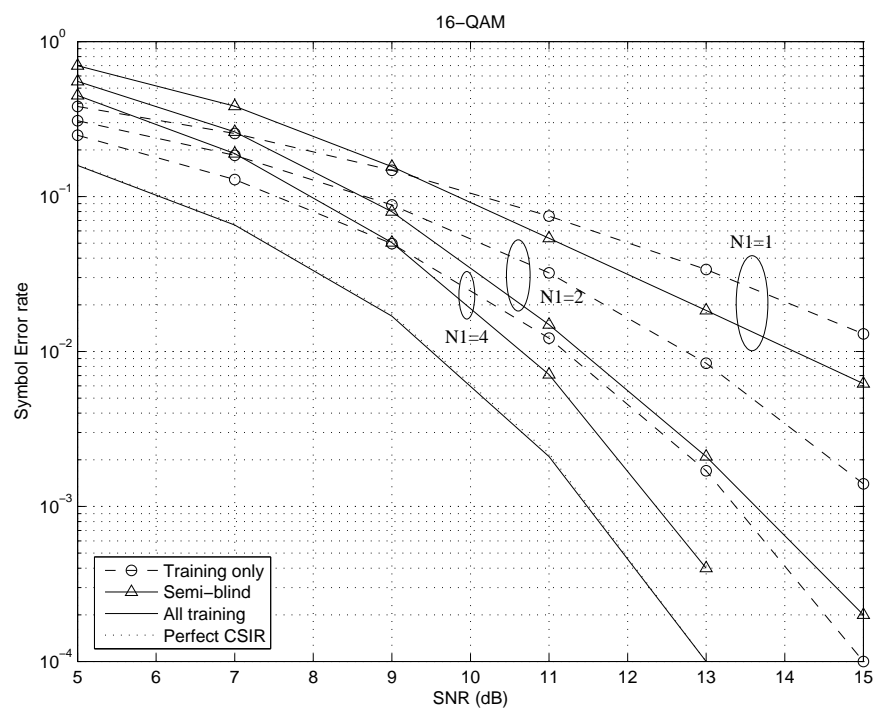

Fig. 1. Comparison between TBO and SB approaches for several training sequences length $\left(N_{1}=1,2,4\right)$ - 16-QAM.

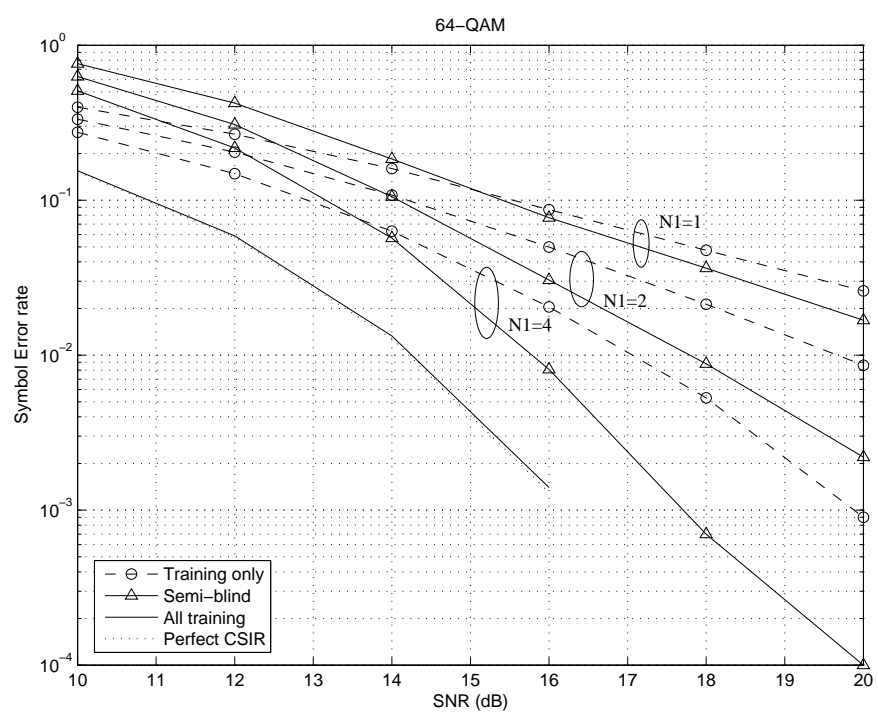

Fig. 2. Comparision between TBO and SB approaches for several training sequences length $\left(N_{1}=1,2,4\right)$ - 64-QAM. 
approach. Interestingly, SB outperforms TSBO even for $P_{e}$ as large as $10^{-1}$. Moreover, this analysis can be extended to any linear processing (synchronization, ....).

\section{REFERENCES}

[1] T.Y. Al-Naffouri, D. Toumpakaris, A. Bahai, and A. Paulraj. An adaptive semi-blind algorithm for channel identification in ofdm. In Signals, Systems and Computers, 2001. Conference Record of the Thirty-Fifth Asilomar Conference on, volume 2, pages 921 - 925, Nov. 2001.

[2] G.B. Giannakis and C. Tepedelenlioglu. irect blind equalizers of multiple FIR channels: a deterministic approach. Signal Processing, IEEE Transactions on [see also Acoustics, Speech, and Signal Processing, IEEE Transactions on], 47:62 - 74, 1932.

[3] IEEE Std 802.16-2004 Revision of IEEE Std 802.16-2001. Ieee standard for local and metropolitan area networks part 16: Air interface for fixed broadband wireless access systems. Technical report, IEEE, 2004.

[4] A. Patel and S. Haykin. Efficient nonlinear blind interference cancellation for DS/CDMA. In Adaptive Systems for Signal Processing, Communications, and Control Symposium, pages 402 - 407, Oct. 2000.

[5] J. Proakis. Digital Communications. McGrawHill, fourth edition, 2000.

[6] V. Radionov and S. Mayrargue. Semi-blind approach to second order identification of simo-fir channel driven by finite-alphabet sequence. In Digital Signal Processing Proceedings, 1997. DSP 97., 1997 13th International Conference on, pages 115 - 118, July 1997. 